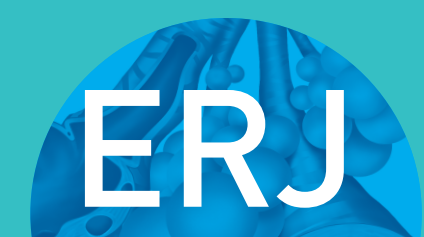

open research
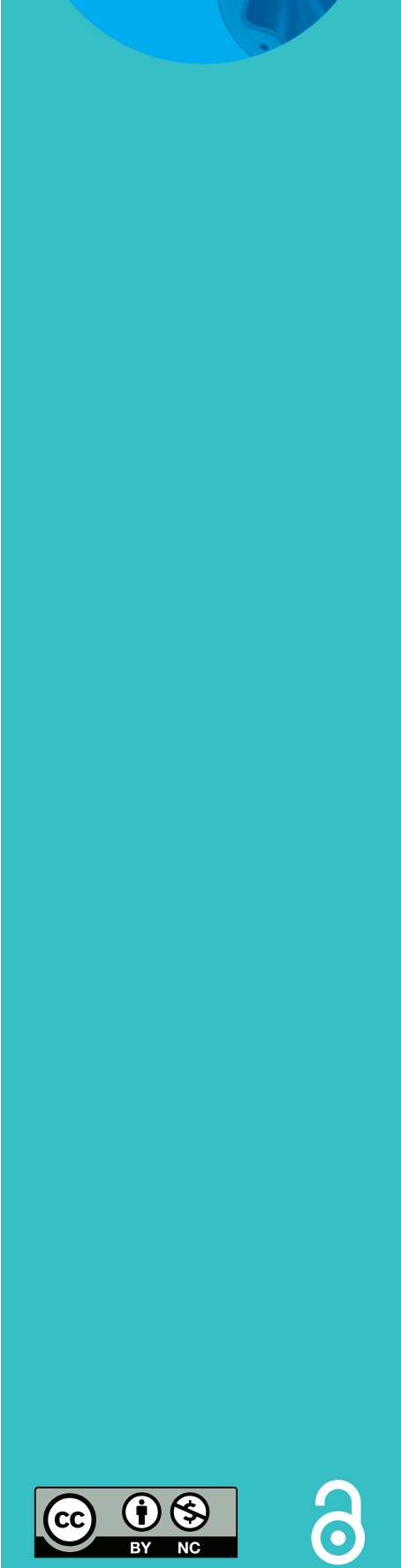

\section{Gaps in care of patients living with pulmonary fibrosis: a joint patient and expert statement on the results of a Europe-wide survey}

Catharina C. Moor ${ }^{1,17}$, Marlies S. Wijsenbeek ${ }^{1,17}$, Elisabetta Balestro ${ }^{2}$, Davide Biondini $^{2}$, Benjamin Bondue ${ }^{3}$, Vincent Cottin $\mathbb{0}^{4}$, Ron Flewett ${ }^{5,18}$, Liam Galvin $^{6,18}$, Steve Jones ${ }^{7,18}$, Maria Molina-Molina ${ }^{8}$, Lurdes Planas-Cerezales ${ }^{8}$, Antje Prasse ${ }^{9}$, Helmut Prosch (10 ${ }^{10}$, Anne-Marie Russell (1) ${ }^{11}$, Michel Viegas ${ }^{12,18}$, Guenther Wanke ${ }^{3,18}$, Wim Wuyts $\mathbb{1}^{14}$, Michael Kreuter ${ }^{15,19}$ and Francesco Bonella ${ }^{16,19}$

\section{ABSTRACT}

Introduction: Pulmonary fibrosis (PF) and its most common form, idiopathic pulmonary fibrosis (IPF), are chronic, progressive diseases resulting in increasing loss of lung function and impaired quality of life and survival. The aim of this joint expert and patient statement was to highlight the most pressing common unmet needs of patients with $\mathrm{PF} / \mathrm{IPF}$, putting forward recommendations to improve the quality of life and health outcomes throughout the patient journey.

Methods: Two online surveys for patients and healthcare professionals (HCPs) were conducted by the European Idiopathic Pulmonary Fibrosis and Related Disorders Federation (EU-IPFF) in 14 European countries. Results: The surveys were answered by 286 patients and 69 HCPs, including physicians and nurses. Delays in diagnosis and timely access to interstitial lung disease specialists and pharmacological treatment have been identified as important gaps in care. Additionally, patients and HCPs reported that a greater focus on symptomcentred management, adequate information, trial information and increasing awareness of PF/IPF is required.

Conclusions: The surveys offer important insights into the current unmet needs of PF/IPF patients. Interventions at different points of the care pathway are needed to improve patient experience.

@ERSpublications

This joint expert and patient statement highlights the most pressing common unmet needs of patients with pulmonary fibrosis, and puts forward recommendations to improve the quality of life and health outcomes throughout the patient journey http://bit.ly/34cTOeo

Cite this article as: Moor CC, Wijsenbeek MS, Balestro E, et al. Gaps in care of patients living with pulmonary fibrosis: a joint patient and expert statement on the results of a Europe-wide survey. ERJ Open Res 2019; 5: 00124-2019 [https://doi.org/10.1183/23120541.00124-2019].

This article has supplementary material available from openres.ersjournals.com

Received: 29 May 2019 | Accepted after revision: 20 July 2019

Copyright $\odot$ ERS 2019. This article is open access and distributed under the terms of the Creative Commons Attribution Non-Commercial Licence 4.0. 


\section{Introduction}

Interstitial lung diseases (ILDs) comprise a diverse collection of more than 200 lung disorders, affecting the interstitium of the lung [1]. A large subgroup of patients with ILD have pulmonary fibrosis (PF); most forms of PF are characterised by a progressive phenotype, are associated with a high burden of disease, and have devastating consequences for patients and their families [2-4]. Idiopathic pulmonary fibrosis (IPF) is the most frequent form and accounts for $17-37 \%$ of all ILDs [5]. A cure for IPF does not currently exist, although there are two approved drugs, pirfenidone and nintedanib, that slow disease progression $[6,7]$. Nonpharmacological treatment options include lung transplantation to prolong life and measures such as pulmonary rehabilitation and supplemental oxygen to ameliorate exercise tolerance and quality of life [8-10].

In 2016, a collaborative effort of patient associations and healthcare professionals (HCPs) was undertaken to gain insights into the needs of patients with IPF, which led to a European IPF Patient Charter [11]. This Charter was presented at the European Parliament to improve awareness and equal access to care around Europe for patients with IPF. We hoped that this would lead to improvements in the care and treatment of patients with fibrotic lung diseases. One of the aims of the current study was to see whether this happened or not. To do so, we aimed to identify the most pressing common unmet needs of patients with $\mathrm{PF} / \mathrm{IPF}$ throughout Europe, and to put forward recommendations in an expert statement to improve quality of life and health outcomes throughout the patient journey.

\section{Participants and methods}

The study was conducted by the European Idiopathic Pulmonary Fibrosis and Related Disorders Federation (EU-IPFF) in association with the European Reference Network on Rare Lung Diseases (ERN-LUNG). This expert statement is a result of the collaboration between patient representatives and medical experts.

Two online surveys were developed: one for PF/IPF patients and one for practising pulmonologists and nurses with ILD expertise. The questions for the surveys were developed by the EU-IPFF working group, consisting of four patient representatives and 14 ILD experts. The group met in person to discuss the topics of the surveys and to reach consensus on the questions. Both surveys contained 62 questions and were circulated between June 29, 2018 and September 8, 2018 in 14 countries. The survey was created in SurveyMonkey (www.surveymonkey.com). An information sheet was developed to inform respondents about the purpose of the project. All respondents were asked to read and understand the terms of the questionnaire and provide their consent. The survey for HCPs was distributed through the ERN-LUNG network and the patient survey was distributed through the EU-IPFF's 17 member organisations via an e-mail that contained a link to the survey. Caregivers were allowed to respond to the survey on behalf of the patient. The surveys are available in the supplementary material.

This study is exempt from ethics review because it consists solely of an online survey that was disseminated to patients via patient groups.

Results have been divided into four geographical subregions: Northern Europe (Denmark, Ireland and UK), Eastern Europe (Bulgaria, Czech Republic and Poland), Southern Europe (Greece, Italy and Spain) and Western Europe (Austria, Belgium, France, Germany and the Netherlands) [12]. Results were collected, tabulated in Excel (Microsoft, Redmond, WA, USA) and bar graphs were generated. Pearson's Chi-squared test was used to compare between geographical subregions. Data were analysed with $\mathrm{R}$ version 3.5.2 (www.r-project.org).

Affiliations: 'Dept of Respiratory Medicine, Erasmus MC, Rotterdam, The Netherlands. ${ }^{2}$ Dept of Cardiac, Thoracic, Vascular Sciences and Public Health, Azienda Ospedaliera di Padova, Università degli Studi di Padova, Padua, Italy. ${ }^{3}$ Dept of Respiratory Medicine, Erasme Hospital, Université Libre de Bruxelles, Brussels, Belgium. ${ }^{4}$ Dept of Respiratory Diseases, Centre Hospitalier Universitaire de Lyon, Lyon, France. ${ }^{5}$ Pulmonary Fibrosis Trust, Lichfield, UK. ${ }^{6}$ Irish Lung Fibrosis Association, Blackrock, Ireland. ${ }^{7}$ Action for Pulmonary Fibrosis, Lichfield, UK. ${ }^{8}$ Respiratory Dept, Hospital Universitari de Bellvitge, Barcelona, Spain. ${ }^{9}$ Dept of Pneumology, Hannover Medical School, Hannover, Germany. ${ }^{10}$ Dept of Biomedical Imaging and Image Guided Interventions, Medical University Vienna, Vienna. Austria. ${ }^{11}$ National Heart and Lung Institute, Imperial College London, London, UK. ${ }^{12}$ Association Belge contre la Fibrose Pulmonaire Idiopathique, Waterloo, Belgium. ${ }^{13}$ Lungenfibrose Forum Austria, Innermanzing, Austria. ${ }^{14}$ Dept of Respiratory Medicine, University Hospitals Leuven, Leuven, Belgium. ${ }^{15}$ Dept of Pneumology and Critical Care Medicine, Universitätsklinikum Heidelberg, Heidelberg, Germany. ${ }^{16}$ Dept of Pneumology and Allergy, Ruhrlandklinik Medical Faculty, University of Duisburg-Essen, Essen, Germany. ${ }^{17}$ These authors share first authorship. ${ }^{18}$ These authors represent the patient's perspective. ${ }^{19}$ These authors share senior authorship.

Correspondence: Marlies S. Wijsenbeek, Dept of Respiratory Medicine, Erasmus MC, University Hospital Rotterdam, 's Gravendijkwal 230, 3015 CE Rotterdam, The Netherlands. E-mail: m.wijsenbeek-lourens@erasmusmc.nl 


\begin{tabular}{|c|c|}
\hline & References \\
\hline Timely and accurate diagnosis & {$[11,13-26]$} \\
\hline More awareness of PF/IPF & {$[11,13-15,18-21,23,26,27]$} \\
\hline Adequate information and education & {$[11,13-19,22-24,26-32]$} \\
\hline Access to pharmacological treatment & {$[11,14,15,19-21,26]$} \\
\hline Interstitial lung disease specialists & {$[11,13-16,18,19,22,25-27]$} \\
\hline Symptom relief & {$[17,18,20,21,23,28,30]$} \\
\hline Psychological support & {$[11,13-15,17,23,26-28]$} \\
\hline More involvement and support of partners & {$[14,16,17,24,28,32,33]$} \\
\hline $\begin{array}{l}\text { Nonpharmacological management } \\
\text { (i.e. supplemental oxygen, pulmonary rehabilitation) }\end{array}$ & {$[11,13,15-17,19,21-23,26,30]$} \\
\hline Access to a multidisciplinary team & {$[11,16,17]$} \\
\hline End-of-life care & {$[11,16,19,20,22,23,26-30]$} \\
\hline
\end{tabular}

In addition, a literature search was conducted for articles about the care pathway and unmet needs of patients with PF/IPF. PubMed and Embase were searched for articles published between January 2010 and March 2018, using the Medical Subject Headings (MeSH) terms "idiopathic pulmonary fibrosis", "pulmonary fibrosis", "interstitial lung disease" or "diffuse parenchymal lung disease" in combination with "care pathway", "unmet needs" and/or "barriers". The search was limited to adults and articles published in English. The reference lists of articles were manually screened for additional publications. Relevant articles were included in order to create an overview of the state of knowledge on the care pathway and unmet needs of patients with PF/IPF. Results of the literature search will be used to compare gaps in care from previous research with the results of the current study.

\section{Results}

\section{Literature search}

The literature search retrieved 1111 articles, of which 966 articles were excluded based on title and abstract. After full-text screening of the remaining articles and exclusion of studies without relevant information on unmet needs and the care pathway in PF/IPF, 22 studies were included (see the supplementary material for details). Unmet needs reported by patients and caregivers were extracted from these studies and are presented in table 1 [11, 13-33].

\section{Survey results}

Respondent characteristics

The patient survey was completed by 286 individuals from 14 different countries, of whom $79 \%$ were patients and $21 \%$ were caregivers (figure 1). The majority of patients had IPF (86\%) and $14 \%$ of

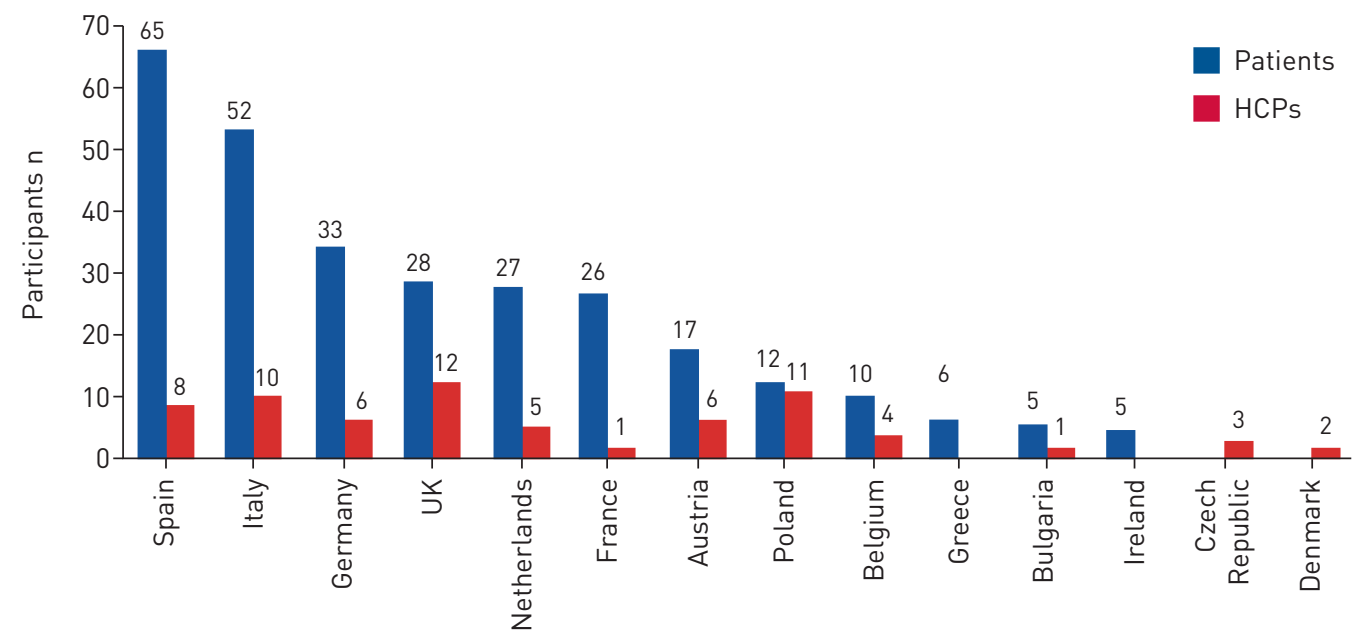

FIGURE 1 Geographical distribution: number of participants (patients and healthcare professionals (HCPs)) per country. 


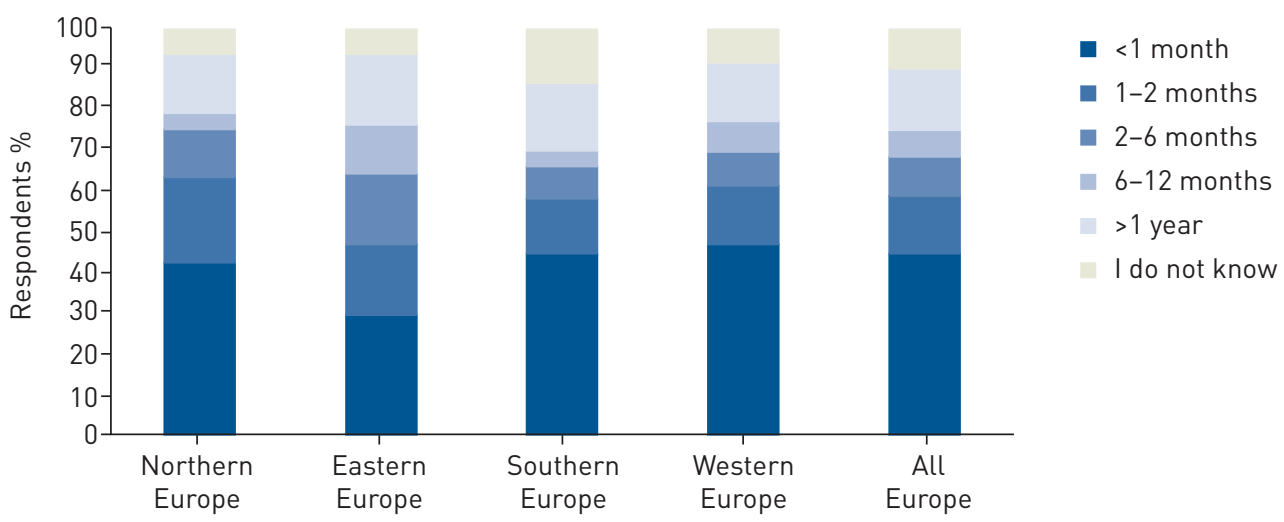

FIGURE 2 Time for referral from general practitioner to a pulmonary physician (patient survey).

respondents had another type of PF. Patients reported diagnosis between 1987 and 2018. The mean age of patients was 66 years and $70 \%$ were male. A fifth of respondents $(21 \%)$ reported a history of PF/IPF in their families.

The questionnaire for HCPs was completed by 69 respondents: 56 physicians (81\%) and 13 specialist nurses (19\%). Most HCPs (87\%) were specialised in ILD and worked at recognised centres of expertise. There was a large variation in the reported number of patients with PF/IPF treated per centre (range 5-3000 patients). The estimated total number of patients managed per year among all participating centres collectively was 10000-11000 for IPF and 27000-28000 for other forms of PF.

Referral pathways and access to ILD specialist care

In order to assess the delay in access to a pulmonary physician, patients were asked to indicate how much time passed before their general practitioner (GP) referred them to a respiratory doctor. Almost half of patients $(45 \%)$ reported that referral took place within 1 month. In contrast, time to referral was $>1$ year for $16 \%$ of patients. No evident differences in referral time were found across Europe $(p=0.84$ ) (figure 2). Furthermore, $33 \%$ of patients reported that their referral to a specialist centre took $<1$ month, with $20 \%$ reporting a wait of $>1$ year. Fewer than half of patients (47\%) reported that a referral to a specialist centre was (very) easy to obtain, whereas $20 \%$ considered it a (very) difficult process.

More than a third of PF/IPF patients (37\%) reported at least one other diagnosis prior to being diagnosed with $\mathrm{PF} / \mathrm{IPF}$. Half of these patients indicated that $>1$ year passed before they were correctly diagnosed (figure 3).

The vast majority of HCPs (94\%) reported that there was access to a multidisciplinary team (MDT) for all $\mathrm{PF} / \mathrm{IPF}$ patients in their centre, but the composition of the MDT varied greatly. In the patient survey, $58 \%$ of respondents stated that diagnosis had been confirmed in an MDT meeting. However, it is unknown if all patients were aware that their case was evaluated by an MDT. Around two-thirds of HCPs (65\%)

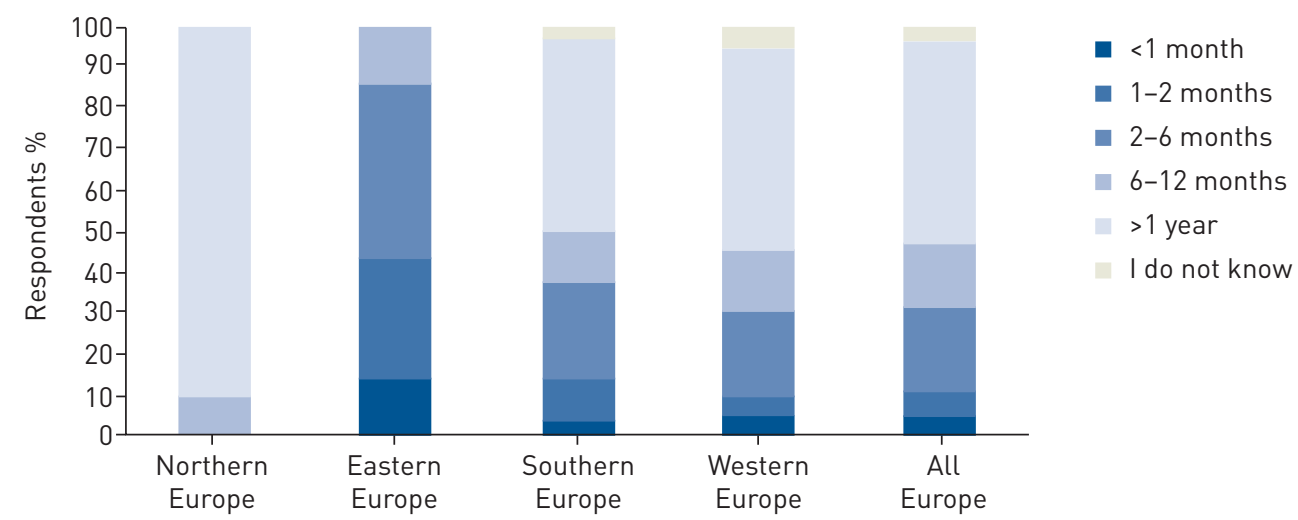

FIGURE 3 Time from initial diagnosis to diagnosis of pulmonary fibrosis/idiopathic pulmonary fibrosis (patient survey). 


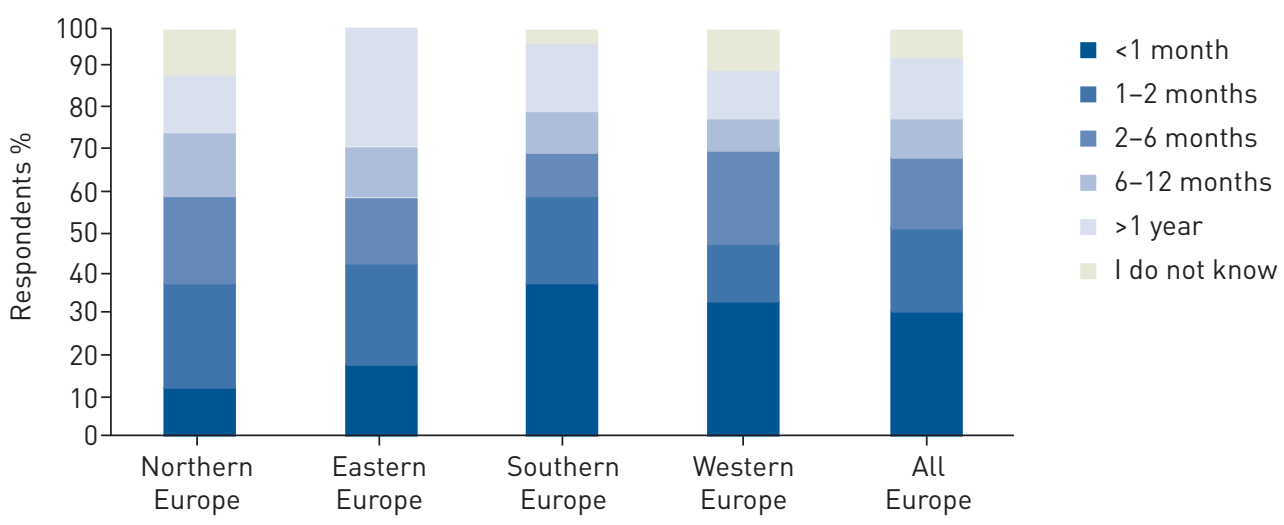

FIGURE 4 Time from diagnosis to start of antifibrotic treatment (patient survey).

answered that ILD specialist nurses were available in their centre, while $52 \%$ of PF/IPF patients responded that they had access to specialist nurses.

Reported access to genetic screening varied. Half of the participating HCPs (49\%) stated that genetic screening was offered, either in their own centre or via referral to another centre. In total, $16 \%$ of surveyed patients underwent genetic testing; of these 45 patients, $42 \%$ stated that they did not receive enough information about their results of the genetic tests.

Access to pharmacological treatment for IPF patients

Both of the approved treatments for IPF, i.e. nintedanib and pirfenidone, were available in all participating countries. Almost all HCPs (93\%) confirmed that antifibrotic drugs could be prescribed in their centres. The majority of respondents with IPF (82\%) were treated with either nintedanib or pirfenidone at the time of the survey.

The time from diagnosis to initiation of treatment varied greatly and this was reflected throughout Europe (figure 4). No statistical differences were found between subregions ( $\mathrm{p}=0.16)$. Although antifibrotic treatment was initiated $<1$ month after diagnosis in $31 \%$ of patients, more than a quarter of patients $(26 \%)$ reported that they had to wait $>6$ months before antifibrotic treatment was started. HCPs reported reimbursement restrictions as the main reason for this delay; $78 \%$ of respondents confirmed that reimbursement restrictions for prescription of antifibrotic treatment exist in their country. In some countries, antifibrotic drugs are only reimbursed when patients are diagnosed in an ILD specialist centre, and lung function and/or age criteria exist in other countries. Specific lung function criteria were identified as the main barrier for prescription of antifibrotic medication by $70 \%$ of HCPs.

Access to nonpharmacological treatment

Almost all HCPs (97\%) were able to prescribe oxygen therapy for PF/IPF patients. More than three-quarters of patients (78\%) reported full coverage for the costs of ambulatory oxygen therapy and two-thirds of patients (64\%) reported full coverage for the costs of oxygen at home.

The vast majority of HCPs $(88 \%)$ could refer patients for pulmonary rehabilitation. A third of HCPs answered that pulmonary rehabilitation was not fully reimbursed in their country. Fewer than half of patients (42\%) stated that they had access to outpatient pulmonary rehabilitation; $11 \%$ of patients also had access to inpatient pulmonary rehabilitation. Just over half of HCPs (58\%) reported that their patients had access to psychological support at their centre, with full reimbursement for $70 \%$ of patients. Patients were not specifically questioned about access to psychological support; however, $10 \%$ of patients spontaneously reported the need for (better) psychological support throughout their disease course.

The most reported eligibility criteria for lung transplantation concerned age and general health condition. Most HCPs (96\%) reported that all eligible patients were referred for lung transplant. In one of the surveyed countries, lung transplantation was not possible at the time of the survey.

Access to palliative care

Of the surveyed patients, $29 \%$ confirmed access to palliative care and $36 \%$ answered that they were involved in palliative care decisions. The majority of HCPs $(88 \%)$ stated that they discussed possibilities for end-of-life care with patients and almost all HCPs (93\%) could prescribe (palliative) medication for symptom relief. 
HCPs were asked to explain at which point in the disease course they initiate palliative care for their patients. Around a third of HCPs answered that palliative care was started at an early stage of the disease if desired by patients. Most HCPs reported that palliative care was initiated in more advanced stages of PF/ IPF. A fifth of HCPs stated that palliative care was only initiated at the end of life.

\section{Communication and education}

The majority of patients $(60 \%)$ had a positive experience while discussing their diagnosis with the pulmonary physician. However, a fifth of patients answered that they did not receive any information about their disease at the time of diagnosis. $73 \%$ of patients and $60 \%$ of HCPs felt that there was enough time to discuss diagnosis and treatment options. Only 39\% of HCPs reported that they received training on how to effectively communicate information on diagnosis and treatment of PF/IPF with their patients.

Three-quarters of patients received a treatment plan following their diagnosis, which was clearly explained in $73 \%$ of cases. Less than a third of patients $(31 \%)$ were involved in development of their treatment plan; this involvement was mostly related to the selection and dosage of antifibrotic medication, initiation of nonpharmacological management and participation in clinical trials.

Patients were asked to give recommendations on how healthcare staff could work more effectively with them and their caregivers. Many patients answered that they would like to have more time allocated for their questions and concerns, and receive more information about PF/IPF, including practical issues such as reimbursement. Furthermore, patients mentioned the need for timely referral to a specialist centre and more awareness of PF/IPF among GPs, nurses and physicians in community hospitals. Around two-thirds

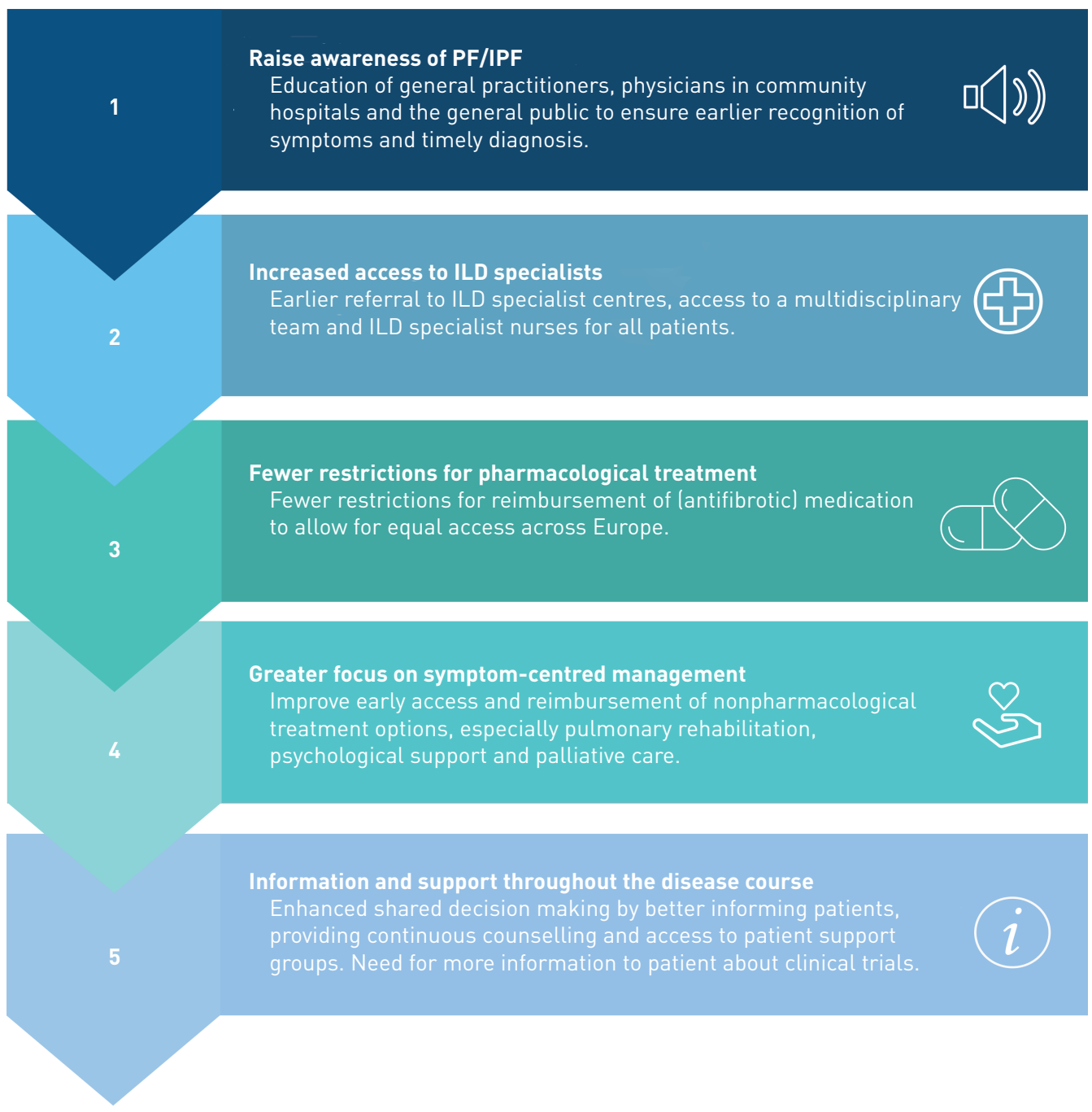

FIGURE 5 General recommendations to improve the pulmonary fibrosis (PF)/idiopathic pulmonary fibrosis (IPF) patient journey. ILD: interstitial lung disease. 
of participating centres (65\%) offered educational activities specifically for PF/IPF patients, such as nurse-led education sessions, information meetings, eHealth programmes and patient support groups. Among the surveyed patients, 39\% attended educational sessions in their treating centre.

Involvement in research

The majority of HCPs (95\%) reported that their centre participated in clinical trials and that they informed their PF/IPF patients of ongoing clinical trials. Half of patients (53\%) were aware of ongoing clinical trials, $31 \%$ had been asked to participate and $25 \%$ had actually participated in a clinical trial. Patient registries for IPF and PF existed in $75 \%$ and $48 \%$ of centres, respectively. A quarter of patients (27\%) declared that they contributed to the collection of registry data.

\section{General recommendations}

In general, $61 \%$ of patients described that their experience with the healthcare system was either good or excellent. Both patients and HCPs were asked about suggestions to improve the patient experiences at different stages of the disease. Based on the answers to this question, five recommendations were proposed by the expert panel (figure 5).

\section{Discussion}

This is the first study investigating unmet needs of patients with PF/IPF in a Europe-wide survey. Despite recent advances in PF/IPF care and research, the unmet needs and gaps in care revealed in this study are in line with previous research (table 1) [11, 13-33].

\section{Referral pathways and access to specialist care}

One of the major unmet needs in PF/IPF care is a timely and accurate diagnosis $[13,15,19,24,25]$. In the current study, a significant number of patients received another diagnosis prior to being diagnosed with PF/IPF; time from initial diagnosis to diagnosis of PF/IPF was often $>1$ year. This is in agreement with previous studies, which showed that many patients receive at least one misdiagnosis, consult more than three physicians before receiving a final diagnosis and have a delay in diagnosis of $>1$ year $[15,25,26,34]$. Although the current study shows less delay than some previous reports, one out of five patients in this study still had to wait $>1$ year for referral to an ILD specialist centre. It is of utmost importance to reduce delays in diagnosis and referral, since previous research indicated that a lengthy diagnostic trajectory can have an adverse effect on quality of life and that delayed access to tertiary referral centres is associated with a higher risk of death in IPF $[13,25,35]$. In fact, access to ILD specialist centres may increase the perceived quality of care $[15,26]$. Access to MDTs appears to have increased in recent years. In contrast to the European IPF Patient Charter in 2016 [11], almost all HCPs in the current study reported access to an MDT, although the composition of the MDT still varies widely.

One of the reasons for delayed diagnosis is the lack of knowledge regarding PF/IPF among the general public, GPs and physicians in community hospitals $[5,11,15,26]$. Improving knowledge about IPF, through education and awareness campaigns, could facilitate earlier diagnosis and referral $[11,13,34]$. A prior study suggested developing symptom-based algorithms for GPs, to help identify which patients should be referred for further analysis [19].

\section{Pharmacological treatment}

Although antifibrotic medication can be prescribed in all participating countries in this study, timely access to treatment was highlighted as an issue by both patients and HCPs. A recent study found that up to $40 \%$ of patients with a confirmed IPF diagnosis do not receive treatment with antifibrotic medication [36]. Barriers to pharmacological treatment include delayed access to specialist care and reimbursement restrictions [36]. Moreover, a watch-and-wait approach is sometimes preferred in patients with mild or relatively stable disease, despite the fact that the importance of early treatment initiation has been emphasised in recent years [5, 36-39].

Our results show that reimbursement restrictions continue to be an important cause of delayed access to antifibrotic treatment. Treatment delays vary due to different prescription criteria. To ensure equal access to antifibrotic medication across Europe, fewer reimbursement restrictions and uniform criteria acknowledging the patient needs reported in this statement are imperative.

\section{Nonpharmacological treatment}

Nonpharmacological treatment options, such as pulmonary rehabilitation, oxygen therapy, psychological support and lung transplantation, are a vital part of holistic care for patients with PF/IPF [2, 40, 41]. Previous studies demonstrated that nonpharmacological treatment options are not equally available for patients in different European countries [11, 19]. In the current survey, the vast majority of HCPs 
indicated that they could refer patients for lung transplantation and pulmonary rehabilitation, as well as being able to prescribe oxygen therapy. In contrast, fewer than half of the patients reported that they had access to pulmonary rehabilitation. This discrepancy could be due to the fact that pulmonary rehabilitation is often not fully reimbursed, that many patients are unaware that pulmonary rehabilitation programmes exist for PF/IPF and that patients often have to travel long distances for pulmonary rehabilitation [11]. The need for better emotional and psychological support for patients and caregivers has been reported frequently and is underlined by the findings from our study $[11,13,14,17-19,21,23,28,33]$. Nevertheless, reimbursement and access to psychological support for PF/IPF patients remains restricted. If referral to a psychologist is not possible, other options for emotional support should be explored. Previous work shows that many patients also benefit from psychological and emotional support through peer support groups, pulmonary rehabilitation and ILD specialist nurses [11, 13, 14, 23, 30, 42, 43]. Strikingly, only half of the surveyed patients in this study had access to ILD specialist nurses, demonstrating that more specialist nurses should be trained.

\section{Access to palliative care}

As present there are no (international) guidelines on palliative care in PF/IPF. This leads to underuse of and varying access to palliative care across Europe, which is also influenced by differences in local resources, cultural and religious beliefs, and misconceptions about the meaning of palliative care $[2,11]$. It is important to acknowledge that palliative care comprises more than just end-of-life care alone and aims to improve quality of life during the whole disease course $[2,44,45]$. Still, our results indicate that many HCPs in Europe start palliative care in more severe stages of PF/IPF. The majority of HCPs in this study stated that they discuss end-of-life care with all patients. However, the optimal timing of end-of-life discussions and referral to palliative care services remains difficult in PF/IPF $[16,19,29]$ and depends on various factors, including culture, religion, etc. Prior reports suggest that early palliative care can potentially reduce symptom burden for patients with IPF, but needs to be tailored to the preferences of individual patients $[2,28]$. Hence, palliative care should be an integral part of comprehensive care for patients with PF/IPF [2].

\section{Communication and education}

Education plays an important role in the management of PF/IPF. To enable shared decision making and enhance communication, patients must be well informed about their disease and its prospects [40, 46]. While our results show that three-quarters of patients receive a treatment plan after their diagnosis, only a third of patients are actually involved in developing this plan. Possible reasons are the lack of time to discuss treatment plans with patients and the fact that patients need to be better educated to become more involved [46]. Adequate information about PF/IPF, more education and continuous counselling were among the frequently reported suggestions for improvement of the care pathway in the patient survey. The need for more information is in agreement with findings from previous surveys and interviews [11, 15-18, 23, 24, 27-29]. Whereas two-thirds of centres in the current study offer education for patients, only a minority of patients attended any educational activity. This suggests that greater awareness of the educational activities among patients may be required or that some patients might prefer to receive written information and/or use online resources $[24,31,46]$. To improve experiences for patients and caregivers, educational material about PF/IPF needs to be easily accessible, understandable, updated frequently and adapted to individual patient's needs $[14,23,24,46]$.

\section{Involvement in research}

Results of this study highlight that patients should be better informed about clinical trials and patient registries. Only half of patients were aware of ongoing clinical trials and only a quarter actually participated in a trial. Previous research suggested that many patients wish to be informed about possibilities to participate in clinical trials and that patients treated in specialist centres were more likely to be participating in a clinical trial $[13,15,27,37]$. Moreover, one study reported that patients who participated in a clinical trial were more hopeful regarding treatment than other patients [13]. Efforts should therefore be made to inform all PF/IPF patients about clinical trials and to refer patients to specialist centres for participation in trials. Many countries have local or national registries for PF/IPF; however, only a quarter of patients indicated that they contribute data to a registry. Improved collaboration with patients and between countries is needed to collect data and establish a multinational registry. Such a registry will not only enhance understanding of disease behaviour, but may also provide insights to improve care and outcomes for patients with PF/IPF $[47,48]$.

\section{Limitations}

This study has several limitations. First, the results are only representative of the situation in the 14 EU-IPFF member countries; newer EU Member States, in particular, have been under-represented. 
Moreover, the HCP survey was distributed through the ERN-LUNG network. This resulted in a high number of responses from physicians in ILD specialist centres, representing an important bias. Similarly, the patients who participated in the survey may have better access to information and specialist care, because they were recruited via support groups. There may also have been a bias towards less impaired patients among the respondents, which makes it difficult to compare the answers of HCPs and patients. Further limitations of this online survey distributed via patient member organisations were a self-reported diagnosis and an unknown response rate.

\section{Conclusions}

This survey and literature search offers important insights into the current unmet needs of PF/IPF patients in Europe and should be considered for healthcare decisions. Recommendations set out in this statement could provide a useful tool for HCPs and policy makers to improve the patient journey and overall care of these rare diseases. Better international collaboration between clinicians, researchers, patients, caregivers, industry partners and governments should be established to solve unmet needs, improve outcomes and develop evidence-based multidisciplinary care for PF/IPF patients.

Acknowledgements: The European Idiopathic Pulmonary Fibrosis and Related Disorders Federation (EU-IPFF) conducted the two surveys in collaboration with the ILD Core Network of the European Reference Network on Rare Lung Diseases (ERN-LUNG). The authors would like to thank all the respondents of the surveys for their valuable input and EU-IPFF members for having supported the dissemination of the questionnaire through their channels.

Support statement: This study was financially supported by Boehringer Ingelheim International GmbH and F. Hoffmann-La Roche Ltd, who had no influence over the content or reporting of the results. Funding information for this article has been deposited with the Crossref Funder Registry.

Conflict of interest: C.C. Moor has nothing to disclose. M.S. Wijsenbeek reports grants and other fees paid to her institution by Hoffmann-La Roche and Boehringer Ingelheim, and other fees paid to her institution by Galapagos, outside the submitted work. E. Balestro has nothing to disclose. D. Biondini has nothing to disclose. B. Bondue reports grants and personal fees from Hoffmann-La Roche and Boehringer Ingelheim outside the submitted work. V. Cottin reports consultancy fees, lecture fees and travel expenses to medical meetings from Actelion and Boehringer Ingelheim, consultancy fees from Bayer/MSD, personal fees for membership of an adjudication committee from Gilead, consultancy fees and lectures fees from Novartis, consultancy fees, lecture fees, travel to medical meetings and a grant to his institution from Roche, speaker fees from Sanofi, personal fees for acting as the chair of a data and safety monitoring board (DSMB) from Promedior, personal fees for membership of DSMBs from Celgene and Galecto, and personal fees for consultancy and membership of a DSMB from Galapagos, outside the submitted work. R. Flewett has nothing to disclose. L. Galvin has nothing to disclose. S. Jones takes part in the annual Patient Advocacy Group meeting organised by Boehringer Ingelheim, who pay a nominal amount to Action for Pulmonary Fibrosis (APF). He organises some meetings with patients for which small honoraria are paid to APF by Boehringer Ingelheim. He has also arranged meetings for Galapagos, Roche, GSK and Vicore with patients, for which an honorarium was sometimes paid to APF M. Molina-Molina reports grants and personal fees from Roche, Boehringer and Esteve-Teijin, grants from GSK, and personal fees from Pfizer, outside the submitted work. L. Planas-Cerezales has nothing to disclose. A. Prasse reports personal fees and nonfinancial support from Boehringer Ingelheim, Roche, AstraZeneca, Novartis, SanofiAventis and NittoDenko, and personal fees from AbbVie and Pliant, outside the submitted work. H. Prosch reports grants and personal fees from Boehringer Ingelheim, and personal fees from Roche, during the conduct of the study. A-M. Russell reports expenses and hospitality to attend scientific advisory board meetings from EU-IPFF, personal fees from ILFA, grants and nonfinancial support from Hoffmann-La Roche and nonfinancial support from Boehringer Ingelheim during the conduct of the study; and grants from Pulmonary Fibrosis Trust UK and nonfinancial support from the ILD Interdisciplinary Network outside the submitted work. M. Viegas has nothing to disclose. G. Wanke has nothing to disclose. W. Wuyts reports grants pay to his university by Roche and Boehringer Ingelheim outside the submitted work. M. Kreuter reports personal fees from Galapagos, and grants and personal fees from Roche and Boehringer Ingelheim, outside the submitted work. F. Bonella reports grants, personal fees and nonfinancial support from Boehringer Ingelheim, Roche and Savara Pharma, outside the submitted work.

\section{References}

1 Travis WD, Costabel U, Hansell DM, et al. An official American Thoracic Society/European Respiratory Society statement: update of the international multidisciplinary classification of the idiopathic interstitial pneumonias. Am J Respir Crit Care Med 2013; 188: 733-748.

2 Kreuter M, Bendstrup E, Russell AM, et al. Palliative care in interstitial lung disease: living well. Lancet Respir Med 2017; 5: 968-980.

3 Lederer DJ, Martinez FJ. Idiopathic pulmonary fibrosis. N Engl J Med 2018; 378: 1811-1823.

4 Kreuter M, Swigris J, Pittrow D, et al. The clinical course of idiopathic pulmonary fibrosis and its association to quality of life over time: longitudinal data from the INSIGHTS-IPF registry. Respir Res 2019; 20: 59.

5 Aiello M, Bertorelli G, Bocchino M, et al. The earlier, the better: impact of early diagnosis on clinical outcome in idiopathic pulmonary fibrosis. Pulm Pharmacol Ther 2017; 44: 7-15.

6 King TE Jr, Bradford WZ, Castro-Bernardini S, et al. A phase 3 trial of pirfenidone in patients with idiopathic pulmonary fibrosis. N Engl J Med 2014; 370: 2083-2092.

7 Richeldi L, du Bois RM, Raghu G, et al. Efficacy and safety of nintedanib in idiopathic pulmonary fibrosis. $N$ Engl J Med 2014; 370: 2071-2082.

8 Dowman L, Hill CJ, Holland AE. Pulmonary rehabilitation for interstitial lung disease. Cochrane Database Syst Rev 2014; 10: CD006322. 
9 Visca D, Mori L, Tsipouri V, et al. Effect of ambulatory oxygen on quality of life for patients with fibrotic lung disease $(\mathrm{AmbOx})$ : a prospective, open-label, mixed-method, crossover randomised controlled trial. Lancet Respir Med 2018; 6: 759-770.

10 Garibaldi BT, Danoff SK. Symptom-based management of the idiopathic interstitial pneumonia. Respirology 2016; 21: $1357-1365$

11 Bonella F, Wijsenbeek M, Molina-Molina M, et al. European IPF Patient Charter: unmet needs and a call to action for healthcare policymakers. Eur Respir J 2016; 47: 597-606.

12 United Nations Statistics Division. Standard Country or Area Codes for Statistical Use (M49). 2019. https:// unstats.un.org/unsd/methodology/m49 Date last accessed: September 3, 2019.

13 Duck A, Spencer LG, Bailey S, et al. Perceptions, experiences and needs of patients with idiopathic pulmonary fibrosis. J Adv Nurs 2015; 71: 1055-1065.

14 Russell AM, Ripamonti E, Vancheri C. Qualitative European survey of patients with idiopathic pulmonary fibrosis: patients' perspectives of the disease and treatment. BMC Pulm Med 2016; 16: 10.

15 Schoenheit G, Becattelli I, Cohen AH. Living with idiopathic pulmonary fibrosis: an in-depth qualitative survey of European patients. Chron Respir Dis 2011; 8: 225-231.

16 Sampson C, Gill BH, Harrison NK, et al. The care needs of patients with idiopathic pulmonary fibrosis and their carers (CaNoPy): results of a qualitative study. BMC Pulm Med 2015; 15: 155.

17 Overgaard D, Kaldan G, Marsaa K, et al. The lived experience with idiopathic pulmonary fibrosis: a qualitative study. Eur Respir J 2016; 47: 1472-1480.

18 Lindell KO, Kavalieratos D, Gibson KF, et al. The palliative care needs of patients with idiopathic pulmonary fibrosis: a qualitative study of patients and family caregivers. Heart Lung 2017; 46: 24-29.

19 Thickett DR, Kendall C, Spencer LG, et al. Improving care for patients with idiopathic pulmonary fibrosis (IPF) in the UK: a round table discussion. Thorax 2014; 69: 1136-1140.

20 Bridges JF, Paly VF, Barker E, et al. Identifying the benefits and risks of emerging treatments for idiopathic pulmonary fibrosis: a qualitative study. Patient 2015; 8: 85-92.

21 US Food and Drug Administration. The Voice of the Patient: Idiopathic Pulmonary Fibrosis. 2015. www.fda.gov/ media/91396/download Date last accessed: September 3, 2019.

22 British Lung Foundation. Lost in the System. IPF: The Patient Experience in England. 2015. www.blf.org.uk/sites/ default/files/BLF-IPF-Report-2015-Lost-in-the-System-250215.pdf Date last accessed: September 3, 2019.

23 Morisset J, Dube BP, Garvey C, et al. The unmet educational needs of patients with interstitial lung disease. Setting the stage for tailored pulmonary rehabilitation. Ann Am Thorac Soc 2016; 13: 1026-1033.

24 Ramadurai D, Corder S, Churney T, et al. Understanding the informational needs of patients with IPF and their caregivers: "You get diagnosed, and you ask this question right away, what does this mean?". BMJ Open Qual 2018; 7: e000207.

25 Cosgrove GP, Bianchi P, Danese S, et al. Barriers to timely diagnosis of interstitial lung disease in the real world: the INTENSITY survey. BMC Pulm Med 2018; 18: 9.

26 Cottin V, Bourdin A, Crestani B, et al. Healthcare pathway and patients' expectations in pulmonary fibrosis. ERJ Open Res 2017; 3: 00134-2016.

27 van Manen MJ, Kreuter M, van den Blink B, et al. What patients with pulmonary fibrosis and their partners think: a live, educative survey in the Netherlands and Germany. ERJ Open Res 2017; 3: 00065-2016.

28 Bajwah S, Higginson IJ, Ross JR, et al. The palliative care needs for fibrotic interstitial lung disease: a qualitative study of patients, informal caregivers and health professionals. Palliat Med 2013; 27: 869-876.

29 Bajwah S, Koffman J, Higginson IJ, et al. "I wish I knew more ..." the end-of-life planning and information needs for end-stage fibrotic interstitial lung disease: views of patients, carers and health professionals. BMJ Support Palliat Care 2013; 3: 84-90.

30 Holland AE, Fiore JF Jr, Goh N, et al. Be honest and help me prepare for the future: what people with interstitial lung disease want from education in pulmonary rehabilitation. Chron Respir Dis 2015; 12: 93-101.

31 Albright $\mathrm{K}$, Walker T, Baird S, et al. Seeking and sharing: why the pulmonary fibrosis community engages the web 2.0 environment. BMC Pulm Med 2016; 16: 4.

32 Shah RJ, Collard HR, Morisset J. Burden, resilience and coping in caregivers of patients with interstitial lung disease. Heart Lung 2018; 47: 264-268.

33 Belkin A, Albright K, Swigris JJ. A qualitative study of informal caregivers' perspectives on the effects of idiopathic pulmonary fibrosis. BMJ Open Respir Res 2014; 1: e000007.

34 Purokivi M, Hodgson U, Myllarniemi M, et al. Are physicians in primary health care able to recognize pulmonary fibrosis? Eur Clin Respir J 2017; 4: 1290339.

35 Lamas DJ, Kawut SM, Bagiella E, et al. Delayed access and survival in idiopathic pulmonary fibrosis: a cohor study. Am J Respir Crit Care Med 2011; 184: 842-847.

36 Maher TM, Molina-Molina M, Russell AM, et al. Unmet needs in the treatment of idiopathic pulmonary fibrosis - insights from patient chart review in five European countries. BMC Pulm Med 2017; 17: 124.

37 Oldham JM, Noth I. Idiopathic pulmonary fibrosis: early detection and referral. Respir Med 2014; 108: 819-829.

38 Cottin V, Richeldi L. Neglected evidence in idiopathic pulmonary fibrosis and the importance of early diagnosis and treatment. Eur Respir Rev 2014; 23: 106-110.

39 Molina-Molina M, Aburto M, Acosta O, et al. Importance of early diagnosis and treatment in idiopathic pulmonary fibrosis. Expert Rev Respir Med 2018; 12: 537-539.

40 Lee JS, McLaughlin S, Collard HR. Comprehensive care of the patient with idiopathic pulmonary fibrosis. Curr Opin Pulm Med 2011; 17: 348-354.

41 Caminati A, Cassandro R, Torre O, et al. Severe idiopathic pulmonary fibrosis: what can be done? Eur Respir Rev 2017; 26: 170047.

42 van Manen MJG, van 't Spijker A, Tak NC, et al. Patient and partner empowerment programme for idiopathic pulmonary fibrosis. Eur Respir J 2017; 49: 1601596

43 Lindell KO, Olshansky E, Song MK, et al. Impact of a disease-management program on symptom burden and health-related quality of life in patients with idiopathic pulmonary fibrosis and their care partners. Heart Lung 2010; 39: 304-313.

44 Danoff SK, Schonhoft EH. Role of support measures and palliative care. Curr Opin Pulm Med 2013; 19: 480-484. 
45 World Health Organization. WHO Definition of Palliative Care. 2019. www.who.int/cancer/palliative/definition/en Date last accessed: September 3, 2019.

46 Fernandez Perez ER, Zelarney P, Thomas S, et al. An educational initiative to improve the team-based care of patients with idiopathic pulmonary fibrosis. ERJ Open Res 2018; 4: 00093-2017.

47 Ryerson CJ, Corte TJ, Collard HR, et al. A global registry for idiopathic pulmonary fibrosis: the time is now. Eur Respir J 2014; 44: 273-276.

48 Cottin V, Annesi-Maesano I, Günther A, et al. Ariane-IPF: seeking collaboration through the launch of a federation of European registries on idiopathic pulmonary fibrosis. Eur Respir J 2019; 53: 1900539. 